\title{
Dynamic Stress-Deformed States of a Circular Tunnel of Small Position Under Harmonic Disturbances
}

\author{
Khamidulla Sagdiyev ${ }^{1}$, Zafar Boltayev ${ }^{2 *}$, Tulkin Ruziyev ${ }^{3}$, Uktam Jurayev ${ }^{4}$, and Farruh \\ Jalolov $^{5}$ \\ ${ }^{1}$ Institute of Mechanics and Seismic Resistance of Structures of the AS R Uz, Tashkent, \\ ${ }^{2}$ Mathematics Department, Bukhara engineering-technological institute, Bukhara, Uzbekistan \\ ${ }^{3}$ Bukhara medical institute, Bukhara, Uzbekistan \\ ${ }^{4}$ Tashkent Institute of Chemical Technology, Tashkent, Republic of Uzbekistan \\ ${ }^{5}$ Mathematics and Natural Sciences Department, Bukhara branch of the Tashkent Institute of \\ Irrigation and Agricultural Mechanization Engineers, Bukhara, Uzbekistan
}

\begin{abstract}
There are many underground tunnels of various shapes located in seismically active areas that need to be protected from seismic impacts. The paper considers the impact of harmonic waves on a cylindrical shell located in a viscoelastic half-plane. The study's main purpose is to determine the stress-strain state of a cylindrical shell when exposed to harmonic waves. The basic equation of viscoelasticity in displacements with the corresponding boundary conditions is obtained. The problem posed is solved in mixed potentials that satisfy the wave equation with complex parameters. The solution is expressed in terms of special Bessel and Hankel functions. As a result of multiple reflections, a system of algebraic equations with complex coefficients is obtained. In the future, this system is solved by the Gauss method with the selection of the main element. The analytical solution is obtained in infinite series, the convergence of which is investigated numerically. The numerical results were obtained using the MATLAB software package. The reliability of the research results is confirmed by good agreement with theoretical and experimental results and those obtained by other authors.
\end{abstract}

\section{Introduction}

To determine the dynamic stress-strain state (SSS) of underground tunnels and pipelines under the influence of seismic loads, various approaches are used [1-3]. The first approach, the so-called seismodynamic theory of foundations, was developed [4], which calculates underground tunnels and pipelines. The further development of this theory belongs to [5-7]. The second approach for determining the dynamic stress-strain state was proposed [8-10]. A third approach is the wave theory of the foundation, which is used for calculating underground structures, laid down [11]. Another way to determine the seismic stress state of structures of underground structures when calculating them on analog accelerograms is

* Corresponding author: boltayev-z@mail.ru 
associated with the use of wave dynamics methods. In the case of a sufficiently extended cavity and the action directed perpendicular to its longitudinal axis, the medium surrounding the cavity and the lining are under plane deformation, and the problem of determining the stress-strain state of the massif and lining is reduced to the plane problem of the dynamic theory of elasticity (or viscoelasticity). It is known that the length of seismic waves $\lambda$ exceeds the diameters of the cross-sections of the tunnel. Of particular interest is when $\frac{D}{\lambda}<1$. In [11], the problem of stress concentration in an infinite linear - elastic plane near a circular cavity of diameter $\mathrm{D}$ was solved during the propagation of longitudinal harmonic waves of length $\lambda$. The maximum coefficients of dynamic stress concentrations $\mathrm{K}_{\sigma}$ (the ratio of the maximum stresses on the hole contour to the amplitude of the incident plane of the wave) are investigated depending on the values of the parameter $\frac{D}{\lambda}$. At long wavelengths $\left(\frac{D}{\lambda}=0.04: 0.16\right)$, it turned out that the maximum coefficients of dynamic concentrations were $5-10 \%$ higher than static ones.

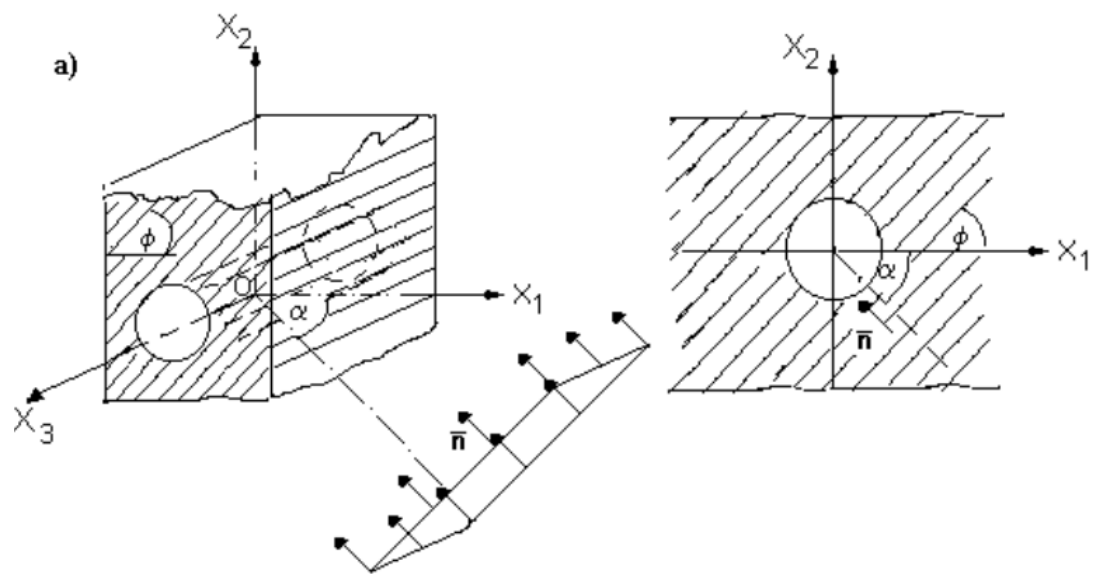

Fig.1. Scheme of the effect of harmonic seismic waves on a cylindrical shell in a viscoelastic halfspace ( $\alpha$ is angle of incidence of the wave, $\vec{n}$ is the unit vector in the direction of propagation of seismic waves).

Further development of the wave problem of underground structures is given in $[12,13]$. Because analytical solutions are limited with simple geometry, simple boundary conditions, and simple behavior of materials, therefore, to calculate the SSS of complex underground structures, numerical methods are used, for example, the finite element method, the boundary element method, the finite difference method and other methods [14,15 ]. In this paper, we consider the dynamic calculations of the stress-strain state of shallowly laid tunnel structures with a circular outline (at a distance of fewer than 5 diameters from the day surface) under the influence of seismic loads.

\section{Methods}

\subsection{Statement of the problem and methods of solution}

Let us consider an isotropic elastic half-space, a circular infinitely extended cylindrical shell of radius $\mathrm{R}$. The shell is characterized by the elastic model $\mathrm{E}_{0}$, Poisson's ratio $v_{0}$, and density $\rho_{0}$. A harmonic seismic load falls on the shell at an angle $\alpha$ in Figure 1 . The problem under consideration is reduced to the problem of plane deformation of the theory 
of viscoelasticity. The equations of motion of a viscoelastic half-space and a circular tunnel in the absence of mass forces have the form [16]:

$$
\tilde{\mu}_{\mathrm{K}} \nabla^{2} \vec{u}_{k}+\left(\tilde{\lambda}_{\mathrm{K}}+\tilde{\mu}_{\mathrm{K}}\right) \operatorname{graddi} \vartheta \vec{u}_{k}=\rho_{\mathrm{K}} \frac{\partial^{2} \vec{u}_{k}}{\partial t^{2}},(\kappa=1,2)
$$

where $\vec{u}_{k}\left(u_{r}, u_{\theta}\right)$ is the vector of displacements of the medium, $\nabla^{2}$ is the Laplace operator, $\rho_{\kappa}$ is the density of the $k$-th body,

$$
\tilde{\lambda}_{\mathrm{K}} f(t)=\lambda_{0 \mathrm{~K}}\left[f(t)-\int_{-\infty}^{t} R_{\lambda_{\mathrm{K}}}(t-\tau) f(\tau) d \tau\right] ; \tilde{\mu}_{\mathrm{K}} f(t)=\mu_{0 \kappa}\left[f(t)-\int_{-\infty}^{t} R_{\mu \mathrm{K}}(t-\tau) f(\tau) d \tau\right]
$$

$f(t)$ is arbitrary function of time $R_{\lambda k}(t-\tau)$ and $R_{\mu k}(t-\tau)$-relaxation kernels of the $\mathrm{k}$-th material, $\lambda_{0 k}, \mu_{0 k}$ are instantaneous module of elasticity of the $\mathrm{k}$-th material, $\mathrm{k}=1,2$. At the contact between the surrounding viscoelastic medium and the circular tunnel, the condition of tight fixation (or sliding) is set:

$$
\begin{aligned}
& \sigma_{r r}^{(k)}=\sigma_{r r}^{(k+1)}, \sigma_{r \theta}^{(k)}=\sigma_{r \theta}^{(k+1)} \\
& u_{r}^{(k)}=u_{r}^{(k+1)}, u_{\theta}^{(k)}=u_{\theta}^{(k+1)}
\end{aligned}
$$

If there is no friction at the contact boundary then

$$
\sigma_{r r}^{(k)}=\sigma_{r r}^{(k+1)}, u_{r}^{(k)}=u_{r}^{(k+1)}, \quad \sigma_{r \theta}^{(k)}=\sigma_{r \theta}^{(k+1)}=0
$$

On the free surface, conditions of stress freedom are set. At infinity, the Sommerfeld radiation conditions are set

$$
\begin{array}{r}
\lim _{r \rightarrow \infty} r \phi_{1}=\text { const }, \quad \lim _{r \rightarrow \infty} r\left(\frac{\partial \phi_{1}}{\partial r}-i \alpha \varphi\right)=0 \\
\lim _{r \rightarrow \infty} r \psi_{1}=\text { const }, \lim _{r \rightarrow \infty} r\left(\frac{\partial \psi_{1}}{\partial r}-i \beta \psi_{1}\right)=0
\end{array}
$$

An incident plane wave is considered to propagate in the positive direction of the $\mathrm{x}$-axis at an angle $\gamma_{0}$ and is represented as follows:

$$
\begin{aligned}
& \phi^{(p)}=\phi_{0} \cos \gamma_{0} e^{i(\alpha x-\omega t)}, \psi^{(p)}=0 \quad \text { - when exposed to longitudinal waves and } \\
& \psi^{(p)}=\psi_{0} e^{i(\beta x-\omega t)} \sin \gamma_{0}, \phi^{(p)}=0 \quad \text { - when exposed to shear waves. }
\end{aligned}
$$

Here and are the magnitudes of the amplitudes; $\alpha$ and $\beta$ are wave numbers, which must be complex numbers $\alpha=\alpha_{R}+i \alpha_{I} ; \beta=\beta_{R}+i \beta_{I}, \alpha_{I}<0$ and $\beta_{I}<0$ denote the damping coefficients; $\alpha_{R}$ and $\beta_{R}$ denote the wave numbers of longitudinal waves and shear waves, respectively. The considered process is harmonic; therefore, no initial conditions are set.

\subsection{Solution techniques}

Let's make the standard transformation in equation (1) as follows. We represent the displacement vector in the form: 


$$
\vec{u}_{k}=\operatorname{grad} \varphi_{k}+\operatorname{rot}\left(\vec{\psi}_{k}\right), \operatorname{div} \vec{\psi}_{k}=0
$$

Here $\varphi_{k}$ and $\vec{\psi}_{k}\left(0, \psi_{k}\right)$ are the longitudinal and transverse potentials, respectively. Substituting (6) into (1) and taking into account that the motion of particles has a steady character, following the principle of superposition, they can be taken into account separately when solving a static problem. Then we obtain, in the case of plane deformation, the following system of wave equations for potentials [17]:

$$
\begin{aligned}
\nabla^{2} \varphi_{k}-\int_{-\infty}^{t}\left[R_{\lambda k}(t-\tau)+2 R_{\mu k}(t-\tau)\right] \nabla^{2} \varphi_{k} d \tau & =\frac{1}{c_{p k}^{2}} \frac{\partial^{2} \varphi_{k}}{\partial t^{2}} \\
\nabla^{2} \psi_{k}-\int_{-\infty}^{t} R_{\mu k}(\mathrm{t}-\tau) \nabla^{2} \psi_{k} d \tau & =\frac{1}{c_{s k}} \frac{\partial^{2} \psi_{k}}{\partial t^{2}}
\end{aligned}
$$

where $\mathrm{c}_{p k}^{2}=\left(\lambda_{k 0}+2 \mu_{k 0}\right) / \rho_{k}, \mathrm{c}_{s k}^{2}=\mu_{k 0} / \rho_{k}, k=1,2$.

We seek the solution to equation (7) in the form:

$$
\phi(r, \theta, t)=\sum_{k=1}^{\infty} \phi_{k}(r, \theta) e^{i \omega t} ; \psi(r, \theta, t)=\sum_{k=1}^{\infty} \psi_{k}(r, \theta) e^{i \omega t}
$$

where $\phi_{\mathrm{K}}(r, \theta)$ and $\psi_{k}(r, \theta)$ are real functions satisfying the equations

$$
\begin{gathered}
\Delta \phi_{k}+\alpha_{L k}^{2} \phi_{k}=0 ; \Delta \psi_{\mathrm{K}}+\beta_{M k}^{2} \psi_{\mathrm{K}}=0 \\
\alpha_{L k}^{2}=\frac{\alpha_{k}^{2}}{1-L_{\mathrm{K}}}, \beta_{M k}^{2}=\frac{\beta_{k}^{2}}{1-\mathrm{M}_{k}}, \\
L_{k}=\int_{0}^{\infty}\left[R_{\lambda}(\xi)+2 R_{\mu}(\xi)\right] \exp (-i \omega \xi) d \xi, M_{k}=\int_{0}^{\infty} R_{\mu}(\xi) \exp (-i \omega \xi) d \xi
\end{gathered}
$$

The study of the interaction and scattering of harmonic waves on a cylindrical body and a free surface is carried out similarly, given in [18]. The incident harmonic wave $\phi^{(p)}$ first falls on the cylindrical shell, and then there is reflection or scattering of the wave: longitudinal $\phi_{1}^{(s)}$ and transverse $\psi_{1}^{(s)}$ Potentials

$$
\Phi_{1}=\phi^{(p)}+\phi_{1}^{(s)}, \Psi_{1}=\psi_{1}^{(s)}
$$

The potentials of longitudinal and transverse waves (10) satisfy the wave equation (9) and boundary conditions (3) - (5). Then the excited wave (10) falls on the half-plane boundary and, as a result,

$$
\Phi=\phi^{(p)}(r, \theta, t)+\sum_{n=1}^{N} \phi_{n}^{(s)}(r, \theta, t) ; \Psi=\psi_{1}^{(s)}(r, \theta, t)+\sum_{n=2}^{N} \psi_{n}^{(s)}(r, \theta, t)
$$

where $\mathrm{n}$ is the number of scattered waves. Expression (11) satisfies boundary conditions (3) - (5) and wave equation (9). Formula (11) considers multiple scattering of waves in a viscoelastic medium from a circular shell.

\subsection{Incident and scattered harmonic waves}

A plane longitudinal wave with potential $\phi^{(p)}$ falls on a cylindrical shell with radius $\mathrm{R}$, the frequency $\omega$ and amplitude of the incident waves $\phi_{0}$ in the form 


$$
\phi^{(p)}=\phi_{0} e^{i(\vec{\alpha} \cdot \vec{r}-\omega t)}, \psi^{(p)}=0
$$

where $\vec{r}=r\left(\cos \theta_{0} \vec{\imath}+\sin \theta_{0} \vec{\jmath}\right), \quad \vec{\alpha}=\alpha\left(\cos \gamma_{0} \vec{\imath}+\sin \gamma_{0} \vec{\jmath}\right), \alpha=\omega / c_{p 1}, \theta_{0}$ - the angle of the cylindrical shell in the main coordinate system, $\gamma_{0}$ is the angle of inclination of the falling loads, $\vec{l}, \vec{j}$ are the unit vectors along the $\mathrm{x}$ and $\mathrm{y}$ axes, respectively (Figure 1). Further, the incident wave $\phi^{(p)}$ in cylindrical coordinates has the form [19].

$$
\phi^{(p)}=\phi_{0} e^{i \alpha_{1} r \cos \gamma_{0}+i \omega t} \sum_{n=-\infty}^{\infty} J_{n}\left(\alpha_{L 1} r\right) e^{i n\left(\theta+\gamma_{0}\right)} .
$$

The scattering of a wave of order $m$ can be expressed in terms of the Hankel functions of the first kind of the nth order of the complex argument

$$
\begin{aligned}
& \phi_{1}^{(s m)}= \sum_{n=-\infty}^{\infty}\left[A_{n}^{s m} \mathrm{H}_{n}^{(1)}\left(\alpha_{L 1} r\right)+\bar{A}_{n}^{s m} \mathrm{H}_{n}^{(2)}\left(\alpha_{L 1} r\right)\right] e^{i n\left(\theta_{0}-\gamma\right)-i \omega t}, \\
& \psi_{1}^{(s m)}=\sum_{n=-\infty}^{\infty}\left[B_{n}^{s m} \mathrm{H}_{n}^{(1)}\left(\beta_{M 1} r\right)+\bar{B}_{n}^{s m} \mathrm{H}_{n}^{(2)}\left(\beta_{M 1} r\right)\right] e^{i n\left(\theta_{0}-\gamma\right)-i \omega t} .
\end{aligned}
$$

Here $A_{n}^{s m}$ and $B_{n}^{s m}$ are the complex scattering coefficients of order $\mathrm{m}, H_{n}^{(1)}$ are the Hankel functions of the first kind of the n-th order of the complex argument. Coefficients, $A_{n}^{s m}, \bar{A}_{n}^{s m}, B_{n}^{s m}, \bar{B}_{n}^{s m}$ are determined from the boundary conditions, which in the considered case have the from:

$$
\begin{aligned}
& \sigma_{r r m}^{(k)}=\sigma_{r r m}^{(k+1)}, \sigma_{r \theta m}^{(k)}=\sigma_{r \theta m}^{(k+1)}, \\
& u_{r m}^{(k)}=u_{r m}^{(k+1)}, u_{\theta m}^{(k)}=u_{\theta m}^{(k+1)} .
\end{aligned}
$$

Similarly, for each incident and scattering harmonic wave, the radiation conditions (5) are satisfied.

The solution to equation (10), for cylindrical shells, is expressed through the Hankel functions of the 1 st and 2 nd kind of the nth order:

$$
\begin{array}{r}
\phi_{2}^{(s)}=\sum_{n=-\infty}^{\infty}\left[C_{n}^{s m} \mathrm{H}_{n}^{(1)}\left(\alpha_{L 2} r\right)+D_{n}^{s m} \mathrm{H}_{n}^{(2)}\left(\alpha_{L 2} r\right)\right] e^{i n\left(\theta_{0}-\gamma\right)-i \omega t}, \\
\psi_{2}^{(s)}=\sum_{n=-\infty}^{\infty}\left[L_{n}^{s m} \mathrm{H}_{n}^{(1)}\left(\beta_{M 2} r\right)+M_{n}^{s m} \mathrm{H}_{n}^{(2)}\left(\beta_{M 2} r\right)\right] e^{i n\left(\theta_{0}-\gamma\right)-i \omega t},
\end{array}
$$

where, $C_{n}^{s m}, D_{n}^{s m}, L_{n}^{s m}, M_{n}^{s m}$ are the expansion coefficients, which are determined by the corresponding boundary conditions; $H_{n}^{(1)}\left(\alpha_{L 2} r\right), H_{n}^{(2)}\left(\alpha_{L 2} r\right), H_{n}^{(1)}\left(\beta_{M 2} r\right)$ and $H_{n}^{(2)}\left(\beta_{M 2} r\right)$ and is the Hankel function of the $1^{\text {st }}$ and $2^{\text {nd }}$ kind of the $\mathrm{n}^{\text {th }}$ order, respectively. Solution (14) satisfies at infinity $\mathrm{r} \rightarrow \infty$ the Sommerfeld radiation condition (5). For this, there must be

$$
\overline{\mathrm{A}}_{n}^{s m}=\bar{B}_{n}^{s m}=0
$$

The solution to equation (14) is represented as: 


$$
\begin{aligned}
\phi_{1}^{(s m)}=\sum_{n=-\infty}^{\infty}\left[A_{n}^{s m} \mathrm{H}_{n}^{(1)}\left(\alpha_{L 1} r\right)\right] e^{i n\left(\theta_{0}-\gamma\right)-i \omega t}, \\
\psi_{1}^{(s m)}=\sum_{n=-\infty}^{\infty}\left[B_{n}^{s m} \mathrm{H}_{n}^{(1)}\left(\beta_{M 1} r\right)\right] e^{i n\left(\theta_{0}-\gamma\right)-i \omega t} .
\end{aligned}
$$

Thus, using (10) and (11), the potentials of longitudinal and transverse waves are determined.

\subsection{Determination of displacement and stress}

The total potential can be determined by superimposing the potentials of the incident and reflected waves (10) - (17). Hence it follows that voltages and displacements can easily be expressed in terms of displacement potentials

$$
\begin{gathered}
u_{r k}=\frac{\partial \phi_{k}}{\partial r}+\frac{1}{r} \frac{\partial \psi_{k}}{\partial \theta} ; u_{\theta k}=\frac{1}{r} \frac{\partial \phi_{k}}{\partial \theta}-\frac{\partial \psi_{k}}{\partial r} \\
\varepsilon_{r r k}=\frac{\partial u_{r k}}{\partial r} ; \varepsilon_{\theta \theta k}=\frac{1}{r} \frac{\partial u_{\theta k}}{\partial \theta}+\frac{u_{r k}}{r} ; \varepsilon_{r \theta k}=\frac{1}{2}\left(\frac{1}{r} \frac{\partial u_{r k}}{\partial \theta}+\frac{\partial u_{\theta k}}{\partial r}+\frac{u_{\theta k}}{r}\right) \\
\sigma_{r r k}=\tilde{\lambda}_{k} \nabla^{2} \phi_{k}+2 \tilde{\mu}_{k}\left[\frac{\partial^{2} \phi_{k}}{\partial r^{2}}+\frac{\partial}{\partial r}\left(\frac{1}{r} \frac{\partial \phi_{k}}{\partial \theta}\right)\right] \\
\sigma_{\theta \theta k}=\tilde{\lambda}_{k} \nabla^{2} \phi_{k}+2 \tilde{\mu}_{k}\left[\left[\frac{1}{r}\left(\frac{\partial \phi_{k}}{\partial r}+\frac{1}{r} \frac{\partial^{2} \phi_{k}}{\partial \theta^{2}}\right)+\frac{1}{r}\left(\frac{1}{r} \frac{\partial \psi_{k}}{\partial \theta}-\frac{\partial^{2} \psi_{k}}{\partial r \partial \theta}\right)\right]\right. \\
\sigma_{z z}=\tilde{\lambda}_{k} \nabla^{2} \phi_{k} ; \sigma_{r \theta k}=2 \tilde{\mu}_{k}\left(\frac{1}{r} \frac{\partial^{2} \phi_{k}}{\partial \theta \partial r}-\frac{1}{r^{2}} \frac{\partial \phi_{k}}{\partial \theta}\right) .
\end{gathered}
$$

where $\varepsilon_{r r}, \varepsilon_{r \theta}, \varepsilon_{\theta \theta}$ are the elements of the strain tensor; $-\sigma_{r r}, \sigma_{r \theta}, \sigma_{\theta \theta}, \sigma_{z z}$ elements of the stress tensor.

To determine arbitrary constants, $\mathrm{A}_{n}^{s m}, B_{n}^{s m}, C_{n}^{s m}, D_{n}^{s m}, L_{n}^{s m}, M_{n}^{s m}$ - used from the boundary conditions (15) and the condition is free from forces on the surface of the halfplane. Then we obtain systems of algebraic equations with complex coefficients with $6 \mathrm{~m}$ unknown quantities and equations. The coefficients are expressed in terms of the special Bessel and Hankel functions. To solve a system of algebraic equations, the Gauss method is used to select the main element. The analytical solution was obtained in infinite series, the convergence of which was investigated numerically [20]. The numerical results were obtained using the MATLAB software package.

\section{Results and Discussion}

Let us consider the problem of wave scattering by an elastic half-plane from circular shells. We assume that the tunnel structure is a steel shell with a radius, $R_{1}=1.75, R_{2}=2.0, h=$ $0.25, \theta_{0}=0^{0}, \mathrm{H} / \mathrm{R}=2.0, \mathrm{~m}=2$. In the calculations, we used the Koltunov-Rzhanitsyn threeparameter relaxation kernel:

$$
R(t)=A e^{-\beta t} / t^{1-\alpha} \text { with the parameters: } A=0,048 ; \beta=0.05 ; \alpha=0.1
$$




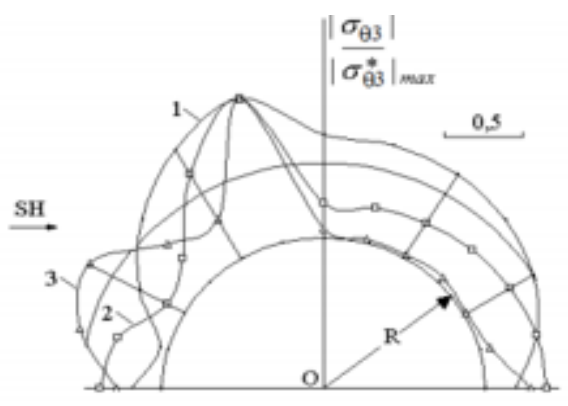

Fig.2. Diagrams of shell contour stress at different frequencies: 1. $\omega=20 \mathrm{~Hz} ; 2 . \omega=40 \mathrm{~Hz}, 3 . \omega=80 \mathrm{~Hz}$

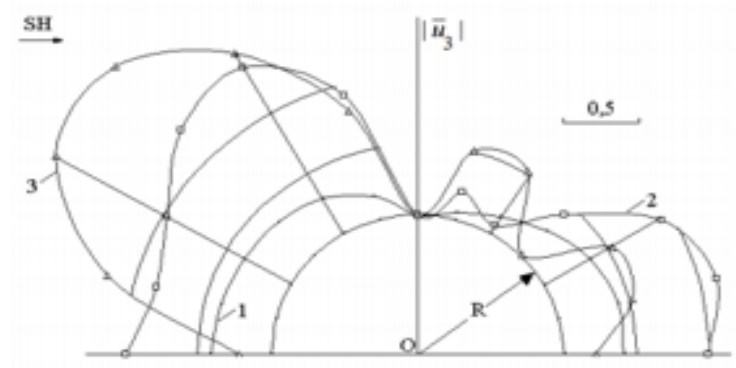

Fig.3. Diagrams of contour displacement of the shell at different frequencies: 1 . $\omega=20 \mathrm{~Hz} ; 2$. $\omega=40 \mathrm{~Hz}$, 3. $\omega=80 \mathrm{~Hz}$

The reliability of the research results obtained is confirmed by good agreement with theoretical and experimental results and those obtained by other authors. The calculation results are shown in Figure 2 and Figure 3 when exposed to shear waves. The maximum loop stresses in the shell are obtained at frequency values

$$
\omega \in[19.30] \mathrm{Hz}
$$

\section{Conclusions}

Regularities of the influence of transverse loads from the action of seismic waves directed normally to the longitudinal axis of the circular tunnel on the stress-strain states of the tunnel are revealed. Dependences of stresses in the tunnel on its diameter, wall thickness, earthquake intensity, rheological properties of the soil have been established. With an increase in the intensity of an earthquake and an increase in the diameter of the tunnel, the voltage changes (increases) as follows: for shells with a diameter of $1000 \mathrm{~mm}$ - by $1,15 \ldots$ 1,25 times, for shells with a diameter of $1500 \mathrm{~mm}$ - by $1,20 \ldots 1,35$ times, for pipes with a diameter $1700 \mathrm{~mm}-1,55 \ldots$ 1,70 times. Proposals have been prepared for improving the regulatory documents when calculating the shell tunnel in seismic areas, taking into account the transverse seismic effect of the wave along the normal to the longitudinal axis of the pipeline. 


\section{Reference}

1. Napetvaridze Sh G, Gehman A S, Spiridonov B V, Rashidov T R, and others Seismic resistance of main pipelines and special structures of the oil and gas industry (Moscow: Nauka) p. 170. (1980)

2. Rashidov T R, Kuzitsov S V, Mardonov B M and Mirzaev I, Applied problems of sesmodinamics of structures. Book 1. The action of seismic waves on underground pipelines and foundations of structures interacting with the soil environment (Tashkent: "Navruz") p. 268. (2019).

3. Gekhman $\mathrm{A} \mathrm{S}$ and Zainetdinov $\mathrm{Kh} \mathrm{Kh}$, Calculation, design and operation of pipelines throughout the world (Moscow: Stroyizdat) p. 180. (1986)

4. Murzakhanov $\mathrm{G} \mathrm{Kh}$ and Oleinikov S S, Estimation of the residual resource of the pipeline under seismic impact Drilling and oil 4 pp. 19-22. (2008)

5. Denisov G V and Lalin V V, Natural vibrations of buried trunk pipelines under seismic impact Pipeline transport: theory and practice, 4 (38) pp. 14-17, (2013)

6. Teshaev M Kh, Safarov I I, Kuldashov N U, Ishmamatov M R and Ruziev T R, On the Distribution of Free Waves on the Surface of a Viscoelastic Cylindrical Cavity Journal of Vibrational Engineering and Technologies 8 (4 ) pp. 579-585. (2020)

7. Kotlyarevsky V A, Ganushkin V I, Kostin A A, Kostin A I and Larionov V I Civil defense shelters (Designs and calculation M: Stroyizdat) p. 606. (1989)

8. Alexandrov A A, Larionov V I and Gumerov R A, Automated monitoring system for main oil pipelines at seismically hazardous areas Vestnik MGTUim. N.E. Bauman. Ser. Mechanical engineering, 5 (98) pp./ 113-126. (2014)

9. Boltaev Z, Safarov I and Razokov T, Natural vibrations of spherical inhomogeneity in a viscoelastic medium International Journal of Scientific and Technology Research 9 (1) pp. 3674-3680. (2020)

10. Abramyan B L, Arutyunyan N Kh and Birger I A and etc Strength, stability, fluctuations: a reference book in 3 volumes (Moscow: Mashinostroenie) T (1) p. 831 (1968).

11. Ainbinder A.B, Calculation of main and field pipelines for strength and stability (Moscow: Nedra) p. 287. (1991)

12. Mavlanov T, Khudainazarov Sh. Calculation of structural-inhomogeneous multiply connected shell structures with viscoelastic elements, Journal E3S Web of Conferences Vol 97 (04054). pp. 1-9 (2019)

13. Bekchanov F, Ergashev R, Mavlanov T and Oleg Glovatskiy Mathematical model of vibrating air pump unit, E3S Web of Conferences 9705045 (2019)

14. Mavlanov $\mathrm{T}$, Khudainazarov $\mathrm{Sh}$ and Khazratkulov I, Natural Vibrations Of Structurally Inhomogeneous Multi-Connected Shell Structures With Viscoelastic Elements. Modeling and Methods of Structural Analysis IOP Conf. Series: Journal of Physics: Conf. Series 1425 012017. (2020)

15. Mirsaidov M.M, Safarov I.I and Teshaev M.X, Dynamic instability of vibrations of thin-wall composite curvorine viscoelastic tubes under the influence of pulse pressure E3SWebofConferences 164 (14013) pp. 1-12. (2020) 
16. Mirsaidov M, Safarov I, Boltayev Z and Teshaev M, Spread waves in a viscoelastic cylindrical body of a sector cross section with cutouts IOP Conference Series: Materials Science and Engineering 869 (4) 042011. (2020)

17. Khudainazarov Sh, Mavlanov T, Qosimov J, Nurova O, Forced vibrations of high-rise buildings IOP Conf. Series: Materials Science and Engineering 869 pp. 1-13. (2020)

18. Mirsaidov M.M, Safarov I.I, Teshaev M.K and Boltayev Z.I, Dynamics of structural Inhomogeneous coaxial-multi-layered systems cylinder-shells Journal of Physics: Conference Series 1706 (1) 012033. (2020)

19. Mirsaidov M.M, Safarov I.I, and Teshaev M Kh, Dynamics of Structurally Inhomogeneous Lamellar and Shell Mechanical Systems. Part 1 Journal of Applied Mathematics and Physics 7 (10) October 14 (2019)

20. Maiboroda V.P, Safarov I.I and Troyanovskii I.E, Free and forced Oscillations of a System of Rigid bodies on Inhomogeneous Viscoelastic snobbery Soviet Machine Science (English Translation of Mashinovedenie) (3) pp. 25-31. (1983) 